\title{
Emended description of Rickettsia felis (Bouyer et al. 2001), a temperature-dependent cultured bacterium
}

Unité des Rickettsies, CNRS UPRESA 6020, Faculté de Medecine, Université de la Mediterranée, 27 Bd Jean Moulin, 13385 Marseille cedex 05, France

\author{
Bernard La Scola, Sonia Meconi, Florence Fenollar, Jean-Marc Rolain, \\ Véronique Roux and Didier Raoult
}

\begin{abstract}
Author for correspondence: Didier Raoult. Tel: +33 4913243 75. Fax: +33491387772. e-mail: Didier.Raoult@medecine.univ-mrs.fr

On the basis of phenotypic data obtained on the strain Marseille-URRWFXCal ${ }_{2}{ }^{\top}$, isolated from the cat flea Ctenocephalides felis, the description of Rickettsia felis (Bouyer et al., 2001) is emended and Marseille-URRWFXCal ${ }_{2}{ }^{\top}$ is proposed as the type strain of the species. On the basis of polyphasic characterization, especially the inability to grow at temperatures higher than $32{ }^{\circ} \mathrm{C}$ on Vero cells that allow growth of other Rickettsia to at least $35^{\circ} \mathrm{C}$, it is confirmed that this agent, although different from other recognized rickettsial species, is genotypically indistinguishable from bacteria previously detected within cat fleas and provisionally named ELB. Comparison of the phenotypic characteristics previously described for $R$. felis and those observed for the isolate in this study indicated some differences, although concurrent analysis of the two was not possible as no extant isolates of the first isolate of $R$. felis exist.
\end{abstract}

Keywords: Rickettsia felis, ELB agent, rpoB gene, actin polymerization, antibiotic susceptibility

\section{INTRODUCTION}

In 1990, whilst examining the potential of cat fleas (Ctenocephalides felis) to act as vectors for a peculiar form of epidemic typhus in the USA, a Rickettsia-like organism, named the ELB agent, was observed within midgut epithelial cells of a group of cat fleas by electron microscopy (Adams et al., 1990). The taxonomic position of this agent within the genus Rickettsia was confirmed by sequence comparison following the successful amplification of a $17 \mathrm{kDa}$ protein gene fragment from infected flea tissue using PCR incorporating genus-specific primers (Azad et al., 1992). Attempts to isolate the ELB agent were made (Radulovic et al., 1995a, b; Higgins et al., 1996), but it now appears that the recovered culture was contaminated by Rickettsia typhi (Radulovic et al., 1995a, 1996). Before this error was identified, the name Rickettsia felis was informally proposed for the organism following characterization of a suspected isolate recovered by Vero cell culture. This isolate was

The GenBank accession numbers for the sequences of the $3^{\prime}$ end of the Rickettsia felis ompA gene and the R. felis rpoB gene are AF231136 and AF236795, respectively. not deposited in any culture collections and therefore no independent confirmatory analysis or further comparison with other Rickettsia species has been possible. The proposed name $R$. felis has recently been formally validated on the basis of molecular data (Bouyer et al., 2001) and confirmed previous studies that underlined the unique position of this Rickettsia species (Adams et al., 1990; Azad et al., 1992; Bouyer et al., 2000; Higgins et al., 1996; Noden et al., 1998; Radulovic et al., 1995b; Raoult et al., 2000; Zavala-Velazquez et al., 2000).

By inoculating crushed cat fleas onto XTC-2 cells incubated at $28{ }^{\circ} \mathrm{C}$, we isolated an intracellular bacterium genotypically indistinguishable from the ELB agent (Raoult et al., 2000), but that was clearly phenotypically distinct from $R$. felis. We present herein additional data for an extended description of Marseille-URRWFXCal ${ }_{2}{ }^{\mathrm{T}}$, the reference strain of the species, and an emended description of the species R. felis.

\section{METHODS}

Source of organisms. Strain Marseille-URRWFXCal ${ }_{2}{ }^{\mathrm{T}}$ was isolated from Ctenocephalides felis obtained from Dr Jay Georgi, Flea Data Inc., Freville, NY, USA. Isolation and 
Table 1. Oligonucleotide primers used for $r p o B$ and $3^{\prime}$ end of ompA PCR amplifications and sequencing reactions

\begin{tabular}{|c|c|c|c|c|}
\hline Gene & Primer & Nucleotide sequence $\left(5^{\prime}-3^{\prime}\right)$ & $\begin{array}{l}\text { Gene position } \\
\text { relative to } \mathrm{ORF}\end{array}$ & Reference \\
\hline \multirow[t]{15}{*}{ omp $A$} & OMPAPUL3519* & GGTATAATAACTGACAATAC & $3519-3538$ & This manuscript \\
\hline & $190-5238^{*}$ & ACTATTAAAGGCTAGGCTATT & $5238-5218$ & Fournier et al. (1998) \\
\hline & OMPAPUL4905* & TCAGGCTCTGAAGTTAAACT & $4905-4924$ & This manuscript \\
\hline & $190-5831 *$ & GTGTCGCTAGGTTTTACAAC & $5831-5812$ & Fournier et al. (1998) \\
\hline & $190-5768^{*}$ & CACCGCTACAGGAAGCAGAT & $5768-5787$ & Fournier et al. (1998) \\
\hline & $190-6808^{*}$ & CACGAACTTTCACACTACC & $6808-6790$ & Fournier et al. (1998) \\
\hline & OMPAPUL3775† & GCTAAGAACGCTGATGTTGA & $3775-3794$ & This manuscript \\
\hline & OMPAPUL3794† & TCAACATCAGCGTTCTTAGC & $3794-3775$ & This manuscript \\
\hline & OMPAPUL4320† & TTATCGTACCGTTACCGGCTG & $4320-4300$ & This manuscript \\
\hline & OMPAPUL4495† & CTGAGATATTTGCTAACGA & $4495-4513$ & This manuscript \\
\hline & OMPAPUL4924† & AGTTTAACTTCAGAGCCTGA & $4924-4905$ & This manuscript \\
\hline & OMPAPUL5569† & GCTGAAGATATAGCTGAAGA & $5569-5588$ & This manuscript \\
\hline & OMPAPUL5588† & TCTTCAGCTATATCTTCAGC & $5588-5569$ & This manuscript \\
\hline & OMPAPUL6249† & CGGCACTAGAGACTGTCGGC & $6249-6268$ & This manuscript \\
\hline & OMPAPUL6322 $\dagger$ & CATTAACTGACCTGTATAGC & $6322-6303$ & This manuscript \\
\hline \multirow[t]{16}{*}{ rров } & RPOPULM25* & GTAATTTTATCAGTYAGGAG & M25-M6 & This manuscript \\
\hline & RPOPUL539* & TGGACTTTTCCTTCATCATG & $539-520$ & This manuscript \\
\hline & Bap355D* & GAGCAAGAAGTATATATGGG & $400-419$ & Drancourt et al. (1999) \\
\hline & RPOPUL2237* & TCTACCTGCTCAACAATACC & $2237-2218$ & This manuscript \\
\hline & RPOPUL1939* & CAAGGAGAGTTYATTAATTGCCG & 1939-1961 & This manuscript \\
\hline & Bap3850r* & GCCCAACATTCCATTTCRCC & $3927-3908$ & Drancourt et al. (1999) \\
\hline & RPOPUL965† & CTGCAAGCGATGAAGTATTA & $965-984$ & This manuscript \\
\hline & RPOPUL984† & TAATACTTCATCGCTTGCAG & $984-965$ & This manuscript \\
\hline & RPOPUL1586† & CCCAATTTATGGATCAAACAAA & $1586-1607$ & This manuscript \\
\hline & RPOPUL1607† & TTTGTTTGATCCATAAATTGGG & $1607-1586$ & This manuscript \\
\hline & RPOPUL2630† & AAGCGTTGCGTCATCTTGAT & $2630-2650$ & This manuscript \\
\hline & RPOPUL2650† & ATCAAGATGACGCAACGCTT & $2650-2630$ & This manuscript \\
\hline & RPOPUL3096† & GGAAGATGCAAATGTAATGAATG & $3096-3118$ & This manuscript \\
\hline & RPOPUL3118† & CATTCATTACATTTGCATCTTCC & $3118-3096$ & This manuscript \\
\hline & RPOPUL3538† & CTTGAGCTATACGGAGAGAA & $3538-3557$ & This manuscript \\
\hline & RPOPUL3557† & TTCTCTCCGTATAGCTCAAG & $3557-3538$ & This manuscript \\
\hline
\end{tabular}

* Oligonucleotide primer used for PCR amplification and sequencing reaction.

$\dagger$ Oligonucleotide primer used only for sequencing reaction.

maintenance of this isolate in XTC-2 cells has been detailed elsewhere (Raoult et al., 2000). The isolate was carefully checked for lack of $R$. typhi contamination (Raoult et al., 2000). Other strains studied were R. typhi (Wilmington strain), Rickettsia prowazekii (Brein 1 strain), Rickettsia conorii (Seven strain) and Rickettsia akarii (MK Kaplan strain). Routine culture of these bacteria in HEL cells has been described previously (La Scola \& Raoult, 1996).

Analysis of major proteins using SDS-PAGE and Westernblotting. Rickettsial strains were renografin-purified as described previously (Raoult et al., 2000). Renografinpurified preparations of Marseille-URRWFXCal ${ }_{2}^{\mathrm{T}}, R$. typhi, $R$. prowazekii, $R$. conorii and $R$. akari proteins were resuspended in SDS-PAGE sample buffer $(0.625 \mathrm{M}$ Tris/ $\mathrm{HCl}, \mathrm{pH} 8.0 ; 2 \%$, w/v, SDS ; $5 \%$, v/v, 2-mercaptoethanol; $10 \%, \mathrm{v} / \mathrm{v}$, glycerol; $0.002 \%, \mathrm{w} / \mathrm{v}$, bromophenol blue). An aliquot of each was then heated for $10 \mathrm{~min}$ at $100{ }^{\circ} \mathrm{C}$, then heated and unheated aliquots were loaded onto on 9$16 \%$ linear gradient polyacrylamide gels $(18 \mathrm{~cm} \times 20 \mathrm{~cm} \times$ $1.5 \mathrm{~mm})$. Proteins were electrophoretically resolved at
$40 \mathrm{~mA}$ for $5 \mathrm{~h}$ at $10^{\circ} \mathrm{C}$ (Laemmli, 1970). Resolved proteins were stained with silver stain. Major immunogenic proteins were studied by Western blotting using purified unheated antigens as previously reported (Laemmli, 1970; Teysseire et al., 1992) and anti-Marseille-URRWFXCal ${ }_{2}{ }^{\mathrm{T}}$ rabbit polyclonal antibodies (Raoult et al., 2000).

Detection of actin polymerization. Adherent XTC-2 cells were cultivated in glass Lab-Tek chamber slides (Miles) under the conditions described above. MarseilleURRWFXCal ${ }_{2}{ }^{\mathrm{T}}$ cells were deposited on adherent cells for 1 or $2 \mathrm{~h}$ incubation at room temperature. Extracellular bacteria were removed by extensive washing of the cells. After $48 \mathrm{~h}$ incubation at $28{ }^{\circ} \mathrm{C}$, cells were fixed with $1 \%$ formaldehyde for $20 \mathrm{~min}$ and washed. Actin filaments and bacteria were stained by incubation with PBS containing $10 \mathrm{U}$ bodipy phallacidin $\mathrm{ml}^{-1}$ (Molecular Probes), $1 \%$ bovine serum albumin, $100 \mu \mathrm{g}$ lysophosphatidylcholine $\mathrm{ml}^{-1}$ (Sigma) and specific mouse antibodies directed against Marseille-URRWFXCal ${ }_{2}{ }^{\mathrm{T}}$ at 1:400 dilution for $30 \mathrm{~min}$. After washing, rhodamine-conjugated $\mathrm{F}\left(\mathrm{ab}^{\prime}\right)_{2}$ antimouse 
IgG was added to the cell preparation for $30 \mathrm{~min}$. Cells were then examined with a laser confocal fluorescence microscope (Leica) equipped with a $\times 100$ (NA 1.4) oil immersion lens. As a control, $R$. conorii and $R$. typhi were processed in the same manner, but using specific polyclonal antibodies for detection of bacteria.

Antibiotic susceptibility. The activity of erythromycin against Marseille-URRWFXCal ${ }_{2}{ }^{\mathrm{T}}$ was determined in Vero cells by a colorimetric assay using Gimenez staining. Vero cells in culture in 48-well microtitre plates were infected with 2000 p.f.u. rickettsiae for $1 \mathrm{~h}$ at room temperature. Erythromycin was added at different concentrations in different rows. Drug-free rows infected with 2000, 200, 20 or 0 p.f.u. served as controls. After 9 days incubation of the plates at $32{ }^{\circ} \mathrm{C}$, cell culture monolayers were stained using Gimenez dye to reveal the presence of infected foci (clusters of rickettsiae) in 25 randomly chosen fields for each well. The minimal antibiotic concentration allowing complete inhibition of foci formation as compared to drug-free control was recorded as the MIC. For doxycycline and rifampicin, a single concentration of $4 \mu \mathrm{g} \mathrm{ml}^{-1}$ was tested. Experiments were performed in duplicate to confirm the results. The incubation temperature was lowered to $32{ }^{\circ} \mathrm{C}$ to allow growth of Marseille-URRWFXCal ${ }_{2}{ }^{\mathrm{T}}$. As controls, $R$. conorii and $R$. typh $i$ were processed in the same manner.

rpoB and ompA $3^{\prime}$ end PCR amplification and sequencing. Genomic DNA extractions, PCR amplifications and sequencing reactions were performed as described previously (Raoult et al., 2000). PCR amplification of the ompA 3' end was obtained using primer pair 190-5768 and 190-6808. An improved PCR method for walking in uncloned genomic DNA, using the Universal Genome Walker kit (Clontech) had to be used for the determination of the $5^{\prime}$ end of the sequence. Primers 190-5831, OMPAPUL5588, OMPAPUL5238, OMPAPUL4924, OMPAPUL4320 and OMPAPUL3794 were used. ompA was then amplified in three fragments using primer pairs OMPAPUL3519 and 1905238, OMPAPUL4905 and 190-5831, and 190-5768 and 190-6808, and sequences were confirmed using the primers listed in Table 1. For rрoB amplification, the primers were designed following identification of suitable hybridization sites within conserved regions of the gene after alignment of the sequence of Bar 29 (AF076436), Rickettsia massiliae (AF76433), $R$. conorii Seven (AF076434), R. prowazekii (AF034531) and R. typhi (AF083622), or were described by Drancourt \& Raoult (1999). rpoB was amplified in three fragments using primer pairs RPOPULM25 and RPOPUL539, Bap355D and RPOPUL2237, and RPOPUL1939 and Bap3850r. Sequencing reactions were performed using the primers described in Table 1. The omp $A$ and rpoB sequences were translated into protein sequences using PC/GENE software (IntelliGenetics). The percentages of similarity were determined using the same software package. The $5^{\prime}$ and $3^{\prime}$ ends of the ompA gene were joined and phylogenetic analyses were performed as described previously (Raoult et al., 2000).

\section{RESULTS AND DISCUSSION}

The SDS-PAGE profile of Marseille-URRWFXCal ${ }_{2}{ }^{\mathrm{T}}$ was clearly different from those derived from $R$. typhi, $R$. prowazekii, $R$. conorii and $R$. akari (Fig. 1). On unheated samples, Marseille-URRWFXCal ${ }_{2}^{\mathrm{T}}$ expressed a high-molecular-mass protein of over $150 \mathrm{kDa}$ which was not shared with other species. On

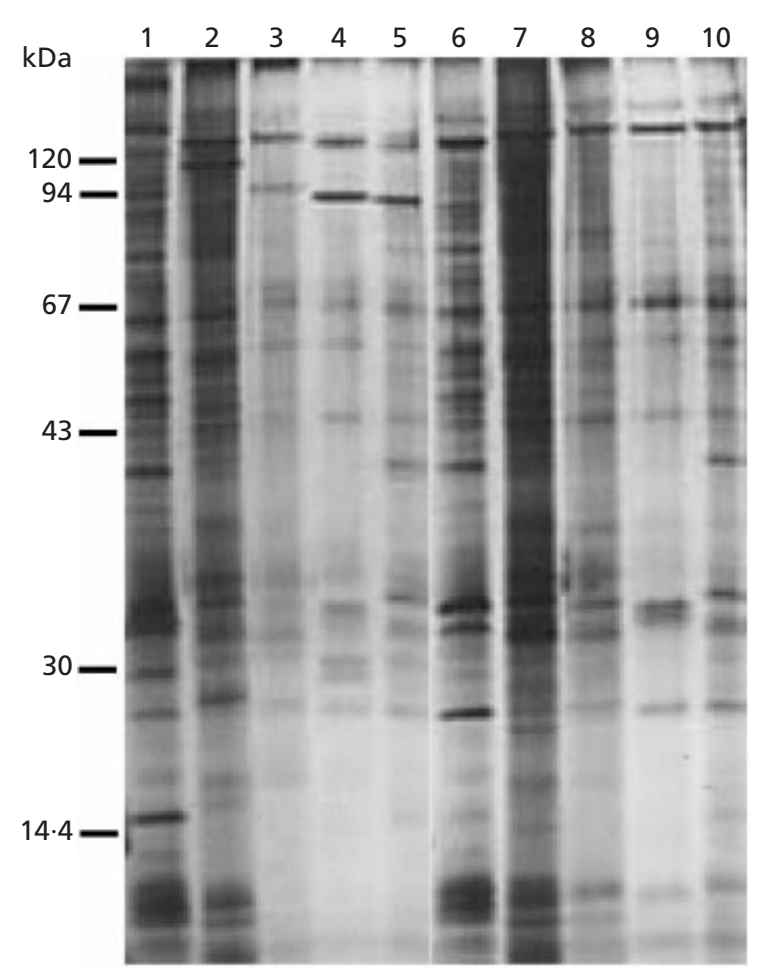

Fig. 1. Silver-stained SDS-PAGE of whole-cell protein preparations of Marseille-URRWFXCal ${ }_{2}^{\top}, R$. conorii, $R$. typhi, $R$. prowazekii and $R$. akari. Lanes: 1,6 , Marseille-URRWFXCal ${ }_{2}{ }^{\top}$; 2, 7, R. conorii; 3, 8, R. typhi; 4, 8, R. prowazekii; 5, 10, R. akari. Lanes: $1-5$, unheated samples; $6-10$, heated samples.

heated samples, all species shared a high-molecularmass protein of about $120 \mathrm{kDa}$. Some other minor differences were also observed, thus the SDS-PAGE protein profile of our strain is markedly different to that of the first description of $R$. felis which was previously found to closely resemble that of $R$. typhi. This resemblance was also reflected by Western blotting (Radulovic et al., 1995b; Higgins et al., 1996; Azad et al., 1997), whereas we recently demonstrated that the immunogenic antigens of MarseilleURRWFXCal ${ }_{2}^{\mathrm{T}}$ were distinguishable from those of $R$. typhi with both a human serum reacting specifically to Marseille-URRWFXCal ${ }_{2}{ }^{\mathrm{T}}$ and a mouse antiserum raised against the purified isolate (Raoult et al., 2000), specifically because of a reaction against the $30 \mathrm{kDa}$ antigen. These findings were confirmed in this study using rabbit polyclonal antibodies (Fig. 2).

Fluorescence and double staining of actin and bacteria was performed to evaluate capability of MarseilleURRWFXCal ${ }_{2}{ }^{\mathrm{T}}$ to polymerize actin intracellularly. Polar polymerization of actin was not observed within cells infected by Marseille-URRWFXCal ${ }_{2}{ }^{\mathrm{T}}$ or $R$. typhi contrary to those infected by $R$. conorii as described previously (Fig. 3). Actin polymerization seems to be associated with a capability to grow in an intranuclear location, a feature common to the spotted fever group rickettsia studied to date, but absent in the typhus 


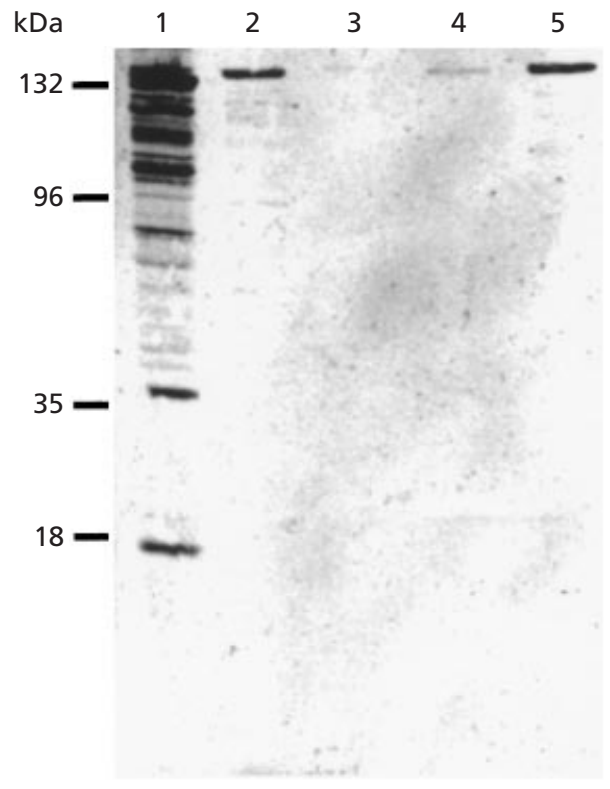

Fig. 2. Western blot of rickettsial proteins probed with anti-Marseille-URRWFXCal ${ }_{2}{ }^{\top}$ rabbit polyclonal serum. Lanes: 1, Marseille-URRWFXCal ${ }_{2}{ }^{2} ; 2, R$. conorii; 3, R. typhi; 4, R. prowazekii; 5, R. akari.

group members and Marseille-URRWFXCal ${ }_{2}^{\mathrm{T}}$ (Heinzen et al., 1993; Teysseire et al., 1992; Burgdorfer et al., 1968; Van Kirk et al., 2000).

Analysis of the antibiotic susceptibilities of MarseilleURRWFXCal ${ }_{2}{ }^{\mathrm{T}}$, revealed an MIC for erythromycin of $32 \mu \mathrm{g} \mathrm{ml}^{-1}$, and that a concentration of $4 \mu \mathrm{g}$ doxycycline or rifampicin $\mathrm{ml}^{-1}$ was inhibitory. Results obtained for controls were identical to those obtained previously (Rolain et al., 1998). Susceptibility to doxycycline and rifampicin is thus common to all species tested, whereas susceptibility to erythromycin is a feature of the typhus group (Rolain et al., 1998) and of the first description of R. felis (Radulovic et al., 1995a, 1996).

The omp $A$ and $r р о B$ genes were sequenced from base 3539 to 6722 and from base 1 to 3866, respectively. For rpoB, sequence similarities ranged from 96 (R. massiliae, Bar $29, R$. conorii) to $87 \%$ for nucleotide sequences. Sequence similarity was $92 \%$ between $R$. felis and $R$. prowazekii. For amino acid sequences, similarities ranged from 98 (R. massiliae, Bar 29, $R$. conorii) to $83 \%$ (R. typhi). Sequence similarity was $96 \%$ between $R$. felis and $R$. prowazekii. The dendrograms obtained from omp $A$ with the three different tree-building methods used showed similar phylogenetic positions for $R$. felis which is clearly separated from other Rickettsia species (bootstrap value of $100 \%$; Fig. 4). These data are in accordance with those reported Bouyer et al. (2001). Interestingly, in their original description of $R$. felis, the authors
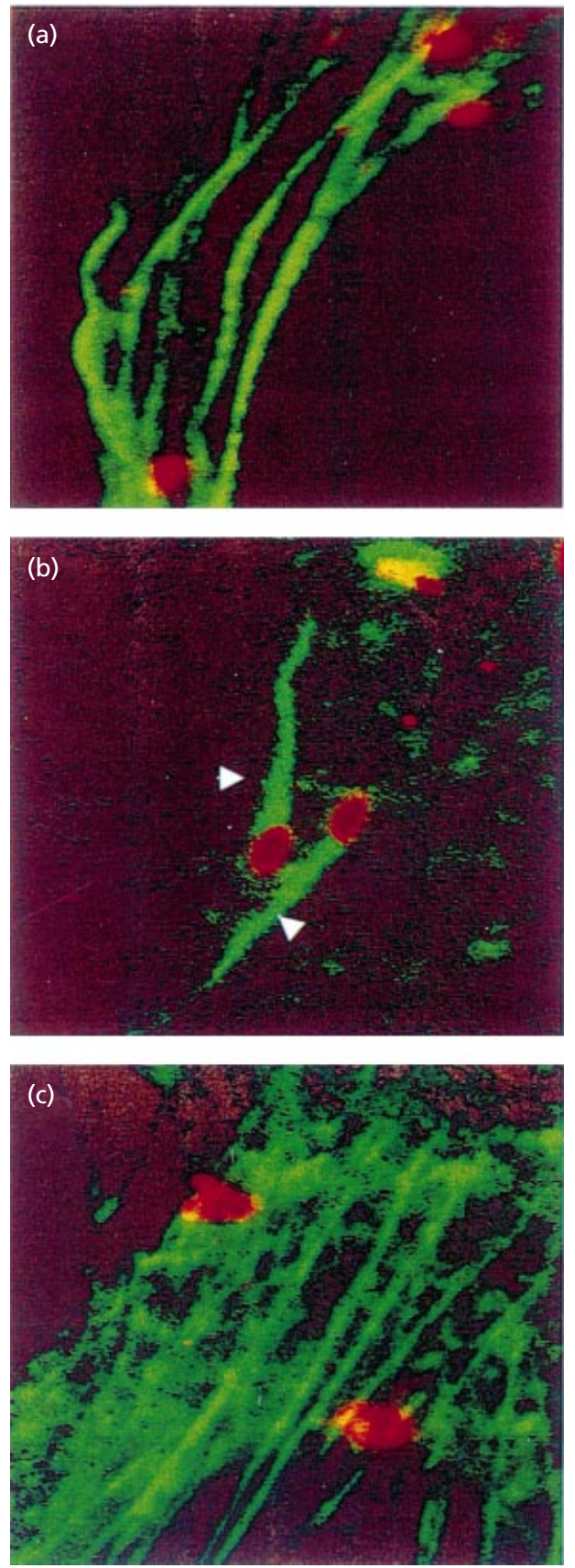

Fig. 3. Double staining of actin and Marseille-URRWFXCal ${ }_{2}^{\top}(a)$, $R$. conorii (b) and $R$. typhi (c). Note the polar actin polymerization within cells infected by $R$. conorii (arrows).

speculated that the rOmpA protein, which is specific to the spotted fever group rickettsia, was absent from their isolate (Radulovic et al., 1995b). However, studies have subsequently demonstrated that the omp $A$ gene in this bacterium contains several premature stops that would result in a truncated rOmpA protein (Bouyer et al., 2000, 2001).

A main observation that differentiates Marseille- 


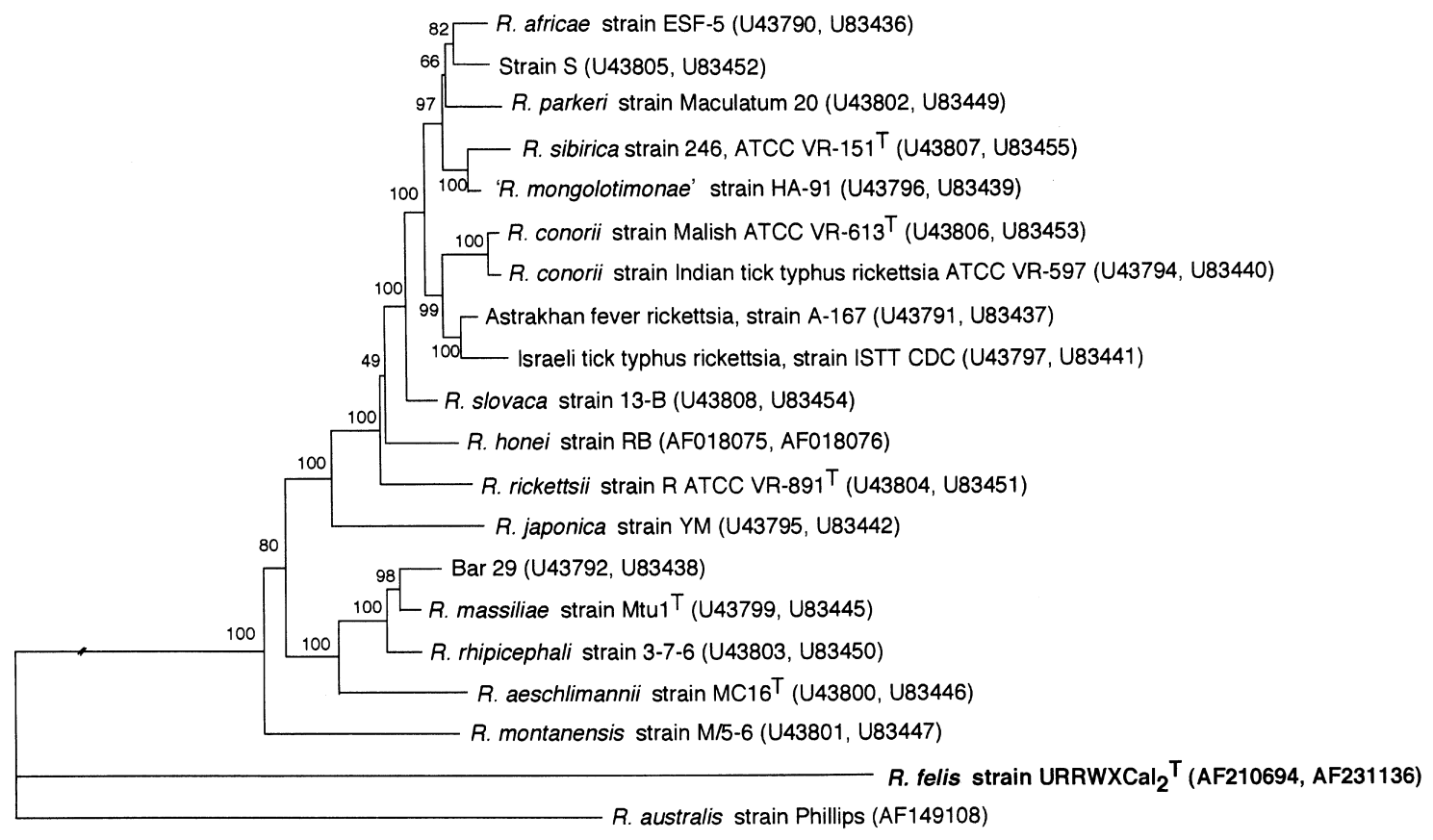

$1.5 \%$ difference

Fig. 4. Phylogenetic tree of the bacteria belonging to the genus Rickettsia, inferred from comparison of ompA sequences. The evolutionary distance values were determined by the neighbour-joining method. The numbers at nodes are proportions of 100 bootstrap resamplings that support the topology shown.

URRWFXCal ${ }_{2}$ Trom previous descriptions of $R$. felis is its growth temperature. Marseille-URRWFXCal ${ }_{2}{ }^{\mathrm{T}}$ did not grow at either 35 or $37^{\circ} \mathrm{C}$ and grew weakly at $32^{\circ} \mathrm{C}$ in Vero cells, whereas it grew well in XTC-2 and Vero cells at $28^{\circ} \mathrm{C}$ (Raoult et al., 2000). As XTC-2 cells degenerate rapidly at temperatures above $28^{\circ} \mathrm{C}$, we could not test the growth of Marseille-URRW$\mathrm{FXCal}_{2}{ }^{\mathrm{T}}$ in these cells at higher temperatures. R. felis has been reported to induce a cytopathic effect leading to the formation of late, tiny plaques when grown at $34{ }^{\circ} \mathrm{C}$ in either Vero cells, HUVECs or L929 cells (Radulovic et al., 1995b). Furthermore, at 32 and $28^{\circ} \mathrm{C}$, Marseille-URRWFXCal ${ }_{2}^{\mathrm{T}}$ did not grow on L929 cells (Raoult et al., 2000). These results demonstrate that the previously isolated $R$. felis strain is not the same organism as that which we repeatedly recovered from infected cat fleas obtained from the El Laboratory colony (Raoult et al., 2000). Although genotypic data derived from PCR-based analysis of the ELB agent in flea extracts concur with those derived from examination of the MarseilleURRWFXCal ${ }_{2}{ }^{\mathrm{T}}$ isolate (Adams et al., 1990; Azad et al., 1992; Bouyer et al., 2000, 2001; Higgins et al., 1996; Noden et al., 1998; Radulovic et al., 1995b; Raoult et al., 2000; Zavala-Velazquez et al., 2000), there is clearly a discrepancy between phenotypic data derived from analysis of the first proposed isolate of the ELB agent, named R. felis, and those of MarseilleURRWFXCal ${ }_{2}{ }^{\mathrm{T}}$. Our inability to address this dis- crepancy resulted directly from the unavailability of extant $R$. felis isolates. Indeed, further doubt over the credibility of data derived from examination of $R$. felis isolates resulted following the isolation of only $R$. typhi from stocks of $R$. felis supplied to our lab by the authors of the original description of this organism (Higgins et al., 1996).

In their recent official description of $R$. felis, Bouyer et al. (2001) justify the formal description of $R$. felis without isolation and description of any type species, as this species is considered as non-cultivable, like Rickettsia peacockii that was also described on the basis of molecular data only (Niebylski et al., 1997). The same approach was also used for the initial description of Tropheryma whipplei, the agent of Whipple's disease (Relman et al., 1992). In the last 2 years, these three micro-organisms have been successfully isolated using different strategies, allowing conventional formal description (Raoult et al., 2000; Simser et al., 2001; La Scola et al., 2001) and demonstrating that 'uncultivable' micro-organisms are mostly 'not yet cultured'. To date there are no human pathogens that cannot be propagated by mean of axenic culture, cell culture or inoculation in animals. Thus when a new potential human pathogen is identified by means of molecular techniques, efforts must be made to propagate it. Moreover, the definition of a species based on genotypic data only, such as $16 \mathrm{~S}$ 
Table 2. Comparison of $R$. felis with other rickettsiae

Data from previous studies (Raoult et al., 2000; Radulovic et al., 1995a, b, 1996; Higgins et al., 1996). NA, Not available.

\begin{tabular}{|c|c|c|c|c|}
\hline Character & R. conorii & R. typhi & $\begin{array}{l}\text { R. felis Marseille- } \\
\text { URRWFXCal }_{2}^{\mathrm{T}}\end{array}$ & 'R.felis' \\
\hline \multicolumn{5}{|l|}{ Growth at: } \\
\hline $28^{\circ} \mathrm{C}(\mathrm{XTC}-2)$ & + & + & + & NA \\
\hline $32{ }^{\circ} \mathrm{C}$ (Vero) & + & + & $+($ weak $)$ & NA \\
\hline $34^{\circ} \mathrm{C}$ (Vero) & + & + & - & + \\
\hline \multicolumn{5}{|l|}{ Growth on: } \\
\hline XTC-2 cells & + & + & + & NA \\
\hline Vero cells & + & + & + & + \\
\hline L-929 cells & + & + & - & + \\
\hline \multicolumn{5}{|l|}{ Observation within: } \\
\hline Cytoplasm & + & + & + & + \\
\hline Nucleus & + & - & - & - \\
\hline $\begin{array}{l}\text { Actin polymerization } \\
\text { in XTC- } 2 \text { cells }\end{array}$ & + & - & - & NA \\
\hline $\begin{array}{l}\text { Inhibition in Vero cells } \\
\text { by } 4 \mu \mathrm{g} \text { erythromycin } \mathrm{ml}^{-1}\end{array}$ & - & + & - & + \\
\hline \multicolumn{5}{|l|}{ Proteins on SDS-PAGE: } \\
\hline $150 \mathrm{kDa}$ & + & - & + & - \\
\hline Heat-labile $30 \mathrm{kDa}$ & - & - & + & - \\
\hline \multicolumn{5}{|l|}{ Detectable genes: } \\
\hline отрA & + & - & + & - \\
\hline omp B & + & + & + & + \\
\hline
\end{tabular}

rDNA, may not be sufficient for defining species, as observed for other species such as Bacillus spp. or Afipia spp. (Ash et al., 1991; Fox et al., 1992; La Scola et al., 2002). To assist the recording of new putative taxa of prokaryotes based on limited genotypic data, it was proposed that such micro-organisms should be named as Candidatus until sufficient data became available to give an official name (Murray \& Schleifer, 1994). Following these recommendations, $R$. felis and $R$. peacockii should have been named 'Candidatus R. felis' and 'Candidatus R. peacockii', awaiting successful cultivation, including stable subcultures, to be proposed for a formal description. Following these recommendations would avoid any confusion such as now observed for $R$. felis for which discrepant phenotypic data will exist in the scientific literature. To cite Murray \& Schleifer (1994), 'it must be hoped that the candidate names used should be validated, but this cannot be guaranteed, and other names might then be more appropriate'. To avoid any confusion which could result from the introduction of a new name after two successive descriptions of $R$. felis (Higgins et al., 1996; Bouyer et al., 2001) we propose to retain the name Rickettsia felis and that strain Marseille$\mathrm{URRWFXCal}_{2}{ }^{\mathrm{T}}$ become the reference strain as the cultivable isolate of the ELB agent. The characters that allow differentiation of $R$. felis from other rickettsial species are summarized in Table 2. Contrary to genotypic data, the phenotypic data about $R$. felis previously reported must now be considered unreliable (Radulovic et al., 1995a, b, 1996; Higgins et al., 1996).

\section{Emendation of the description of Rickettsia felis (Bouyer et al. 2001)}

Rickettsia felis is an obligate intracellular, Gramnegative bacterium in the order Rickettsiales. The reference strain, Marseille-URRWFXCal ${ }_{2}{ }^{\mathrm{T}}$, is available in our official collection and in the French National Culture Collection (Institut Pasteur, Paris, France) under the accession number I-2363. This strain is available upon request form the authors or from the Institut National de la Propriété Industrielle (Paris, France). It was isolated from cat fleas, Ctenocephalides felis, by the shell vial cell culture procedure using XTC2 cells at $28^{\circ} \mathrm{C}$. $R$. felis can be grown in XTC- 2 cells at $28^{\circ} \mathrm{C}$ and Vero cells at 28 and $32^{\circ} \mathrm{C}$, but not on L-929 and MRC- 5 cells or at a higher incubation temperature than $34^{\circ} \mathrm{C}$. R. felis induces cytopathic foci and plaque formation in XTC-2 and Vero cells at 9 and 18 days, respectively. The bacterium is present in the cytoplasm of infected cells and not in the nuclei; it is unable to polymerize actin within the cytoplasm of infected cells. $R$. felis is susceptible to doxycycline and rifampicin and resistant to erythromycin. $R$. felis can be detected in infected cells by Gimenez staining and by immunofluorescence with mouse polyclonal anti- $R$. felis antibody. $R$. felis DNA can be amplified with rickettsial 
specific primer for the $17 \mathrm{kDa}$ protein, 16S rRNA, glt $A$, oтp $A$, omp $B$ and $r p o B$ genes. $R$. felis DNA has been amplified from the blood of a Texas patient suspected of having $R$. typhi infection and from a Brazilian and two Mexican patients with undiagnosed febrile exanthematous illness.

\section{ACKNOWLEDGEMENTS}

The authors are indebted to R. J. Birtles for reviewing the manuscript.

\section{REFERENCES}

Adams, J. R., Schmidtmann, E. T. \& Azad, A. F. (1990). Infection of colonized cat fleas, Ctenocephalides felis (Bouché), with a rickettsia-like microorganism. Am J Trop Med Hyg 43, 400-409.

Ash, C., Farrow, J. A., Dorsch, M., Stackebrandt, E. \& Collins, M. D. (1991). Comparative analysis of Bacillus anthracis, Bacillus cereus, and related species on the basis of reverse transcriptase sequencing of $16 \mathrm{~S}$ rRNA. Int J Syst Bacteriol 41, 343-346.

Azad, A. F., Sacci, J. B., Nelson, W. M., Dasch, G. A., Schmidtmann, E. T. \& Carl, M. (1992). Genetic characterization and transovarial transmission of a typhus-like rickettsia found in cat fleas. Proc Natl Acad Sci U S A 89, 43-46.

Azad, A. F., Radulovic, S., Higgins, J. A., Noden, B. H. \& Troyer, J. M. (1997). Flea-borne rickettsioses: ecologic considerations. Emerg Infect Dis 3, 319-327.

Bouyer, D. H., Crocquet-Valdes, P. A. \& Walker, D. H. (2000). Expression and size determination of the rOmpA protein of Rickettsia felis. In American Society for Rickettsiology-15th Meeting, p. 61. American Society for Rickettsiology and Rickettsial Diseases.

Bouyer, D. H., Stenos, J., Crocquet-Valdes, P. A. \& 7 other authors (2001). Rickettsia felis: molecular characterization of a new member of the spotted fever group. Int J Syst Evol Microbiol 51, 339-347.

Burgdorfer, W., Anacker, R. L., Bird, R. G. \& Bertram, D. S. (1968). Intranuclear growth of Rickettsia rickettsii. J Bacteriol 96, 1415-1418.

Drancourt, M. \& Raoult, D. (1999). Characterization of mutations in rpoB gene in naturally rifampin-resistant Rickettsia species. Antimicrob Agents Chemother 43, 2400-2403.

Fournier, P. E., Roux, V. \& Raoult, D. (1998). Phylogenetic analysis of spotted fever group rickettsiae by study of the surface protein rOmpA. Int J Syst Bacteriol 48, 839-849.

Fox, G. E., Wisotzkey, J. D. \& Jurtshuk, P. J., Jr (1992). How close is close: $16 \mathrm{~S}$ rRNA sequence identity may not be sufficient to guarantee species identity. Int J Syst Bacteriol 42, 166-170.

Heinzen, R. A., Hayes, S. F., Peacock, M. G. \& Hackstad, T. (1993). Directional actin polymerization associated with spotted fever group rickettsia infection of Vero cells. Infect Immun 61, 1926-1935.

Higgins, J. A., Radulovic, S., Schriefer, M. E. \& Azad, A. F. (1996). Rickettsia felis: a new species of pathogenic rickettsia isolated from cat fleas. J Clin Microbiol 34, 671-674.

La Scola, B. \& Raoult, D. (1996). Diagnosis of Mediterranean spotted fever by cultivation of Rickettsia conorii from blood and skin samples using the centrifugation-shell vial technique and by detection of $R$. conorii in circulating endothelial cells: a 6 year follow-up. J Clin Microbiol 34, 2722-2727.

La Scola, B., Fenollar, F., Fournier, P. E., Altwegg, M., Mallet, M. N. \& Raoult, D. (2001). Description of Tropheryma whipplei gen. nov. sp. nov., the Whipple's disease bacillus. Int $J$ Syst Evol Microbiol 51, 1471-1479.

La Scola, B., Mallet, N. M., Grimont, P. \& Raoult, D. (2002). Description of Afipia birgiae sp. nov., Afipia massiliae sp. nov. and recognition of Afipia felis genospecies A. Int J Syst Evol Microbiol 52, 1773-1782.

Laemmli, U. K. (1970). Cleavage of structural proteins during the assembly of the head of bacteriophage T4. Nature 227, 680-685.

Murray, R. G. E. \& Schleifer, K. H. (1994). Taxonomic notes: a proposal for recording the properties of putative taxa of procaryotes. Int J Syst Bacteriol 44, 174-176.

Niebylski, M. L., Schrumpf, M. E., Burgdorfer, W., Fisher, E. R., Gage, K. L. \& Schwan, T. G. (1997). Rickettsia peacockii sp. nov., a new species infecting wood ticks, Dermacentor andersoni, in Western Montana. Int J Syst Bacteriol 47, 446-452.

Noden, B. H., Radulovic, S., Higgins, J. A. \& Azad, A. F. (1998). Molecular identification of Rickettsia typhi and R. felis in co-infected Ctenocephalides felis (Siphonaptera: Pulicidae). J Med Entomol 35, 410-414.

Radulovic, S., Higgins, J. A., Jaworski, D. C. \& Azad, A. F. (1995a). In vitro and in vivo antibiotic susceptibilities of ELB rickettsiae. Antimicrob Agents Chemother 39, 2564-2566.

Radulovic, S., Higgins, J. A., Jaworski, D. C., Dasch, G. A. \& Azad, A. F. (1995b). Isolation, cultivation, and partial characterization of the ELB agent associated with cat fleas. Infect Immun 63, 4826-4829.

Radulovic, S., Higgins, J. A., Jaworski, D. C. \& Azad, A. F. (1996). In vitro and in vivo antibiotic susceptibilities of ELB rickettsiae (erratum). Antimicrob Agents Chemother 40, 2912.

Raoult, D., La Scola, B., Enea, M., Fournier, P. E., Roux, V., Fenollar, F., Galvao, M. A. M. \& De Lamballerie, X. (2000). A flea associated Rickettsia pathogenic for humans. Emerg Infect Dis 7, 73-81.

Relman, D. A., Schmidt, T. M., MacDermott, R. P. \& Falkow, S. (1992). Identification of the uncultured bacillus of Whipple's disease. $N$ Engl J Med 327, 293-301.

Rolain, J. M., Maurin, M., Vestris, G. \& Raoult, D. (1998). In vitro susceptibilities of 27 Rickettsiae to 13 antimicrobials. Antimicrob Agents Chemother 42, 1537-1541.

Simser, J. A., Palmer, A. T., Munderloh, U. G. \& Kurtti, T. J. (2001). Isolation of a spotted fever Rickettsia Rickettsia peacockii in a rocky mountain wood tick Dermacentor andersoni cell line. Appl Environ Microbiol 67, 546-552.

Teysseire, N., Chiche-Portiche, C. \& Raoult, D. (1992). Intracellular movements of Rickettsia conorii and R. typhi based on actin polymerization. Res Microbiol 143, 821-829.

Van Kirk, L. S., Hayes, S. F. \& Heizen, R. A. (2000). Ultrastructure of Rickettsia rickettsii actin tails and localization of cytoskeletal proteins. Infect Immun 68, 4706-4713.

Wedincamp, J., Jr \& Foil, L. D. (2000). Infection and seroconversion of cats exposed to cats fleas (Ctenocephalides felis Bouche) infected with Rickettsia felis. J Vector Ecol 25, 123-126.

Zavala-Velazquez, J. E., Ruiz-Sosa, J. A., Sanchez-Elias, R. A., Becerra-Carmona, G. \& Walker, D. H. (2000). Rickettsia felis rickettsiosis in Yucatan. Lancet 356, 1079-1080. 